\title{
ANÁLISIS SOCIOLINGÜÍSTICO DE LOS REFORMULDORES DE RECTIFICACIÓN EN EL HABLA SANTIAGUINA*
}

\author{
Abelardo San Martín Núñez**
}

\section{Resumen}

En este estudio se analiza el empleo de los reformuladores de rectificación, en una muestra del español hablado en Santiago de Chile, desde el punto de vista sociolingüístico. Específicamente, se describe el comportamiento de los marcadores del discurso, que cumplen la mencionada función, en una muestra estratificada de 120 entrevistas de hablantes santiaguinos, y se establece la distribución sociolingüística de su empleo. Los resultados muestran que, en general, o sea y bueno son los rectificadores más frecuentes, los que son más empleados por los hablantes jóvenes y los del grupo medio alto, respectivamente.

Palabras clave: marcadores del discurso, reformulación, rectificación, variación discursiva, sociolingüística, español de Chile.

\section{SOCIOLINGUISTIC ANALYSIS OF THE RECTIFICACTION Discourse Markers in the Santiago, Chile speech}

\begin{abstract}
In this study the use of rectification reformulators is analyzed in a sample of Spanish spoken in Santiago, Chile, from a sociolinguistic point of view. Specifically, the behavior of discourse markers that meet this function is described and at the same time the sociolinguistics distribution of this use is established, in a stratified sample of 120 interviews. In general, the results show that $o$ sea and bueno are the most common rectifiers, which are mostly used by younger speakers and the upper-middle group, respectively.
\end{abstract}

Keywords: discourse markers, reformulation, rectification, discursive variation, sociolinguistics, Chilean Spanish.

Recibido: 30-01-2015

Aceptado: 02-09-2015

* La realización de este artículo contó con el apoyo del Concurso U-Apoya-Ayuda de Viaje de la Vicerrectoría de Investigación y Desarrollo de la Universidad de Chile. En este trabajo se sintetizan los resultados más relevantes de una de las secciones de nuestra tesis doctoral inédita, "Variación sintáctica y discursiva en el español hablado en Santiago de Chile. Análisis sociolingüístico del queísmo, el dequeísmo, el discurso referido y los marcadores de reformulación", dirigida por el Dr. Humberto López Morales.

** Chileno. Doctor en Filología Hispánica de la Universidad de Valladolid. Académico de la Universidad de Chile, Santiago, Chile. asmartin@uchile.cl 


\section{Introducción}

\subsection{Naturaleza, alcance y objetivos del estudio}

Los estudios sobre los denominados "marcadores del discurso" han coincidido en destacar su importancia para la producción y la comprensión del discurso tanto oral como escrito en diferentes lenguas (cf. Schiffrin, 1987 y 2003; Anscombre y Ducrot, 1994; Martín Zorraquino y Montolío, 1998; Martín Zorraquino y Portolés, 1999; Portolés, 2001 y Loureda y Acín, 2010, entre otros). A pesar de dicha importancia, una de las dimensiones menos indagadas, en español, es la variación social y dialectal del uso de las mencionadas partículas (Carbonero y Santana, 2010). En este artículo se analizará el comportamiento pragmático y la distribución social del empleo de los marcadores de reformulación rectificativa en una muestra socialmente estratificada de 120 entrevistas realizadas a hablantes santiaguinos. Específicamente, los objetivos de nuestro estudio son: a) identificar los marcadores que cumplen la función de reformulación rectificativa en la muestra; b) determinar su frecuencia de empleo y c) correlacionar dichas frecuencias con las variables sociodemográficas: sexo, edad y grupo socioeconómico de los informantes.

\section{Marco conceptual}

\subsection{La variación discursiva y el estudio sociolingüístico de los marcadores}

Nuestro estudio se enmarca en las directrices teóricas y metodológicas generales de la sociolingüística variacionista desarrollada por Labov (1983), con las necesarias adaptaciones al estudio de la variación en el ámbito discursivo. Desde este punto de vista, se pone de relieve "el hecho de que la lengua es inherentemente variable y ordenadamente heterogénea y que dicha heterogeneidad no es aleatoria sino reglada, por lo cual se sustituye la regla categórica chomskiana por la regla variable" (Serrano, 2011, p. 29, el énfasis es nuestro)'. Los estudios en el

1 En relación con el concepto de regla variable, Cortés (1998), señala que su inclusión “significa que la gramática ya no dispone únicamente de reglas categóricas y optativas, sino que entre unas y otras se han de situar las llamadas reglas variables, las que descubrimos en aquellos hechos discursivos en que la opción del hablante no es tan arbitraria como cabe pensar, sino que está sometida a la influencia regular de un número variable de factores" (p. 147, nota 5). 
ámbito del variacionismo han demostrado, en general, la influencia de factores sociales como el sexo, la edad, el nivel educacional o el grupo socioeconómico de los hablantes, en conjunto con la de factores internos, en el comportamiento lingüístico ${ }^{2}$. No obstante, la extensión del concepto de variable sociolingüística al estudio de fenómenos más allá del nivel fonético planteó una serie de dificultades, especialmente, a partir del trabajo de Lavandera (1978) donde se cuestionó explícitamente dicha extensión, arguyendo que no estaba garantizado en el estudio de las mencionadas variables el principio de invariabilidad a nivel semántico. A pesar de las diferentes posturas en torno a este debate, en principio, la variación lingüística "definida como el uso alterno de formas diferentes de decir lo mismo, se puede encontrar prácticamente en todos los niveles de la lengua, desde el más concreto (fonético-fonológico) al más amplio (discurso, por ejemplo), pasando por la gramática y el léxico" (Moreno Fernández, 1998, p. 19).

Como en trabajos anteriores (San Martín 2004-2005, 2011 y 2013 y Rojas et al. 2012), sugerimos la posibilidad de aplicar el concepto de "variable lingüística" -en un sentido amplio- al estudio de los marcadores del discurso. En relación con el problema que nos ocupa, Cortés (1998, p. 160) concluye que el estudio variacionista de los marcadores es posible si se cumple el requisito de contrastar las frecuencias de conectores que cumplan las mismas funciones, lo que trae aparejadas no pocas dificultades. Para el análisis sociolingüístico de los marcadores, es preciso que se asocien las características de los informantes con aquellas partículas que desempeñen una función común en el discurso (Cortés, 1998, p. 154). De acuerdo con nuestra perspectiva (San Martín, 2004-2005, p. 213), una vez identificadas las partículas que cumplan una función pragmática equivalente, la aplicación del concepto de variable sociolingüística al estudio de los marcadores del discurso es factible, "puesto que este tipo de formas parece cumplir con las condiciones propuestas en el marco del variacionismo, es decir, consistir en 'formas alternativas de decir lo mismo'”. Por su parte, Carbonero y Santana (2010, p. 516-517) señalan

2 Silva-Corvalán, en este sentido, señala que "la variación lingüística no es aleatoria sino que está condicionada tanto por factores internos al sistema de la lengua como por factores sociales externos a ella" (2002, p. 2). Según este principio, la variación sociolingüística ha sido definida como "la alternancia de dos o más expresiones de un mismo elemento, cuando ésta no supone ningún tipo de alteración o cambio de naturaleza semántica y cuando se ve condicionada por factores lingüísticos y sociales" (Moreno Fernández, 1998, p. 33). 
la carencia de trabajos que aborden la variación espacial y social de los marcadores del discurso, aunque -al mismo tiempo- destacan el interés de esta línea de trabajo para el mejor conocimiento de estas unidades de procesamiento.

\subsection{La reformulación rectificativa y sus marcadores en la lengua española}

Los marcadores del discurso son unidades lingüísticas de enlace de carácter invariable que no ejercen una función sintáctica a nivel oracional y cuya función primordial es orientar las inferencias que se realizan en la comunicación (Portolés, 2001, p. 25-26) ${ }^{3}$. Para el presente estudio, adoptaremos, principalmente, la perspectiva teórica sobre los marcadores del discurso de Martín Zorraquino y Portolés (1999) y Portolés (2001). De acuerdo con estos autores, los marcadores de reformulación "presentan el miembro del discurso que introducen como una nueva formulación de un miembro anterior" (Martín Zorraquino y Portolés, 1999, p. 4121). En este sentido, "el hablante considera que lo ya dicho no transmite satisfactoriamente su intención comunicativa y utiliza un reformulador para presentar el miembro del discurso que lo sigue como una mejor expresión de lo que pretendió decir con el miembro precedente" (Martín Zorraquino y Portolés, 1999, p.4121). La clasificación delos reformuladores propuesta por los mencionados autores incluye los siguientes subtipos: explicativos, rectificativos, de distanciamiento y recapitulativos. Según su función específica, los reformuladores rectificativos "sustituyen un primer miembro, que presentan como una formulación incorrecta, por otra que la corrige o, al menos, la mejora, por ejemplo, mejor dicho, más bien y digo" (Martín Zorraquino y Portolés, 1999, p. 4122).

Asimismo, Cuenca (2003) indica que la reformulación constituye "a discourse function by which the speaker re-elaborates an idea in order to be more specific and facilitate the hearer's understanding of the original, or in order to extend the information previously given [una función

3 Martín Zorraquino (1998, p. 35-51) plantea que, pese a la heterogeneidad de los marcadores del discurso, relativa tanto a las categorías a las que pertenecen como a su distribución en el discurso y a sus funciones pragmáticas, dichas unidades presentan, entre otras, las siguientes propiedades comunes: a) no pueden ser sustituidas por elementos pronominales o deícticos, b) no pueden ser sometidos a interrogación parcial, c) no admiten la coordinación con elementos equifuncionales y d) no pueden ser reemplazados por elementos pronominales que reproduzcan la totalidad, o la parcialidad, de la oración en la que se sitúan. 
discursiva mediante la cual el hablante reelabora una idea para ser más específico y facilitar la compresión del oyente del mensaje original, o para ampliar la información previamente dada]" (Cuenca, 2003, p. 1071, la traducción es nuestra). Por su parte, Garcés (2003 y 2011) destaca que la reformulación es un "procedimiento de organización del discurso que permite al hablante volver sobre un segmento anterior para reinterpretarlo y presentarlo de manera distinta" (2011, p. 90), que responde a cinco motivaciones de los hablantes: explicar, recapitular, reconsiderar, distanciarse o corregir (2003, p. 114). Aplicando los supuestos de la lingüística textual de Gülich y Kotschi (1983 y 1995) y Rossari (1994), además, Garcés (2003 y 2011) distingue entre la reformulación parafrástica, en la que se plantea una equiparación semántica y pragmática entre los segmentos reformulado y reformulador, y la no parafrástica, en la que se propone un distanciamiento de diferente grado (mínimo, medio y máximo) entre ambos segmentos. Así, para esta autora, la reformulación es un "proceso de reinterpretación de un segmento previo, explícito o implícito, que, según la relación entre los miembros y el tipo de marcador empleado, puede ir desde la equivalencia hasta el distanciamiento" (Garcés, 2008, p. 82) ${ }^{4}$. Su clasificación considera cinco tipos de marcadores de reformulación, de acuerdo con las motivaciones señaladas más arriba: explicación, rectificación, recapitulación, reconsideración y separación (Garcés, 2008, p. 86). Por lo que se refiere al tipo de reformulación que nos ocupa, en la rectificación, el segmento reformulador sustituye o corrige el segmento anterior que se considera inadecuado (por ejemplo, mejor dicho, más bien). Con posterioridad, Garcés distingue entre los procesos reformulativos de corrección, esto es, la modificación de un segmento previo, y de rectificación, es decir, su invalidación parcial o total, tanto en textos escritos (2010) como en discursos orales (2011).

En cuanto al estudio de los reformuladores de rectificación en el español de Chile, en Rojas et al. (2012, p. 115-116) se informa el siguiente inventario de marcadores rectificativos, en un corpus de 72 entrevistas sociolingüísticas: o sea, digo, digamos, más bien y es decir, con un predominio abrumador del primero. Por otro lado, en su relevamiento de

4 Según Garcés (2008, p. 67), la capacidad de replantear enunciados previos mediante otros que nos parecen más expresivos o adecuados para nuestra propia intención es una propiedad de la competencia metapragmática de los hablantes. Este aspecto también ha sido destacado por Portolés (2004). 
los marcadores del discurso empleados en 12 entrevistas de la norma culta santiaguina, Valencia (2014b) señala que los reformuladores constituyen el tipo de marcador menos utilizado, entre los que destaca o sea con valor explicativo, y que solo se encontraron casos de más bien como reformulador de rectificación. De todas formas, cabe destacar que la misma autora declara que en su estudio no se relevaron usos idiosincrásicos o dialectales (Valencia, 2014a, p. 7-8), debido a motivaciones contrastivas, por lo que su análisis solo se limitó a identificar el empleo del inventario de marcadores informado en Martín Zorraquino y Portolés (1999). Esto plantea una diferencia de estrategia de indagación fundamental con respecto a la adoptada en el presente trabajo.

\section{Metodología}

\subsection{Corpus}

El corpus que servirá de base para nuestro estudio corresponde a 120 entrevistas sociolingüísticas pertenecientes al corpus del grupo de Estudio Sociolingüístico del Español de Chile (ESECH) ${ }^{5}$. Las entrevistas fueron realizadas a hablantes santiaguinos, entre 2005 y 2011, por los estudiantes en la asignatura de Sociolingüística de los programas de Licenciatura en Lengua y Literatura Hispánicas y Licenciatura en Lengua y Literatura Inglesas de la Universidad de Chile. En la situación de entrevista, los entrevistadores debían tratar de superar la "paradoja del observador" consiguiendo, de esta forma, una muestra significativa de discurso natural grabado (estilo vernacular) de hablantes representativos de la comunidad de habla en estudio (Labov, 1983). El número de entrevistas antes indicado totaliza, aproximadamente, 116 horas de grabación, cuya transcripción fue exhaustivamente revisada, sin exclusión de ninguna de las secciones del instrumento.

\subsection{Población y muestra}

En nuestra investigación se consideró la población constituida por hombres y mujeres de la Región Metropolitana de más de 20 años de

5 El ESECH es un grupo de investigación del Departamento de Lingüística de la Facultad de Filosofía y Humanidades de la Universidad de Chile coordinado por nosotros. 
edad'. El cuestionario se aplicó a una muestra del tipo "muestra por cuotas con afijación uniforme", en la que se divide a la población en estratos o categorías y se asigna una cuota a cada uno de los distintos estratos (López Morales, 1994, p. 58). Por lo que respecta al tamaño de la muestra, hemos optado por conformarla con un número de informantes considerado, en general, como suficiente en la bibliografía sociolingüística (cf. Trudgill y Hernández Campoy, 2007, s. v. representatividad y López Morales, 1994, p. 52). La muestra, así conformada, comprende un total de 120 entrevistas realizadas a igual número de sujetos, distribuidos como se indica en la tabla 1 que mostramos a continuación:

\begin{tabular}{llllllll}
\hline & $20-34$ & & \multicolumn{3}{c}{$35-54$} & y más & \\
\hline & H & M & H & M & H & M & Total \\
\hline Medio alto & 5 & 5 & 5 & 5 & 5 & 5 & $=30$ \\
\hline Medio & 5 & 5 & 5 & 5 & 5 & 5 & $=30$ \\
\hline Medio bajo & 5 & 5 & 5 & 5 & 5 & 5 & $=30$ \\
\hline Bajo & 5 & 5 & 5 & 5 & 5 & 5 & $=30$ \\
\hline & 20 & 20 & 20 & 20 & 20 & 20 & $=120$ \\
\hline
\end{tabular}

Tabla 1. Proporción de sujetos según características sociodemográficas de sexo, edad y grupo socioeconómico

\section{3. Procedimiento de estratificación social utilizado en ESECH}

Para la estratificación de los sujetos que conforman la muestra del estudio se empleó el sistema de adscripción de estatus social utilizado en ESECH que se basa en la asignación del puntaje -que se indica entre paréntesis- a los informantes, de acuerdo con las siguientes variables: a) nivel educacional (3), b) profesión u ocupación (2) y c) comuna de residencia (1). La explicación en detalle de dichas variables se encuentra en Prieto (1995-1996), cuya propuesta fue perfeccionada y actualizada con base en los estudios acerca de la realidad socioeconómica chilena de la

6 Por lo que refiere a los criterios de asignación de estatus de hablante nativo de Santiago de Chile, de acuerdo con Prieto (1995-1996), se aplicaron las siguientes restricciones en la selección de los sujetos: 1) haber nacido y residido en forma ininterrumpida en Santiago, 2) haber residido en forma ininterrumpida en Santiago desde los cinco años de edad y 3) haber nacido en Santiago y haber residido en Santiago la mayor parte de sus vidas, salvo por periodos que sumados no superen los cuatro años en el tramo de 55 años y más y los tres años en el tramo de 35 a 54 años. 
empresa de Investigaciones de Mercado y Opinión Pública (ADIMARK, 2003), la Descripción Básica de los Niveles Sociales realizada por el Instituto Consultor en Comercialización y Mercado (ICCOM, 2005) y la propuesta de estratificación de la Asociación de Investigadores de Mercado (AIM, 2008) 7 . A partir de la asignación de los mencionados puntajes, se definieron cuatro grupos socioeconómicos, definidos según los rangos siguientes: a) Medio alto (MA): 42 -36, b) Medio (M): 35 - 27, c) Medio bajo (MB): $26-18$ y d) Bajo (B): $17-6^{8}$. El intervalo de cada rango responde a la mayor coincidencia con los puntajes que han establecido los estudios sociológicos y de mercado para la clasificación de estratos socioeconómicos, aunque con las debidas adaptaciones, puesto que en esta muestra no se incluyen ni el grupo de la extrema pobreza ni el de la extrema riqueza.

\section{4. Procedimiento analítico}

Nuestro análisis de la función discursiva "reformulación de rectificación mediante marcadores" se concentró en la determinación de las frecuencias de ocurrencia de cada una de las partículas específicas, que la cumplen en las entrevistas que conforman la muestra recopilada. Asimismo, de modo coherente con nuestros objetivos, se observará la incidencia de los siguientes factores sociodemográficos de los hablantes: sexo, edad y grupo socioeconómico. Por lo que se refiere a la descripción de los procedimientos estadísticos aplicados, en este trabajo seguimos las sugerencias e indicaciones de Moreno Fernández (1990), López Morales (1994) y Hernández Campoy y Almeida (2005). El análisis estadístico atenderá a dos niveles de observación: a) en términos descriptivos, según las frecuencias absolutas y los porcentajes de frecuencia de cada rectificador y $b$ ) en términos interpretativos o inferenciales, con base en la comparación entre las medias o tendencias centrales de los marcadores más frecuentes, esto es, de aquellos con más de 25 ocurrencias. El

7 Cabe precisar que el modelo de nivel socioeconómico fue adaptado de estos estudios de mercado en función de los objetivos que se propone el grupo ESECH, que busca definir un perfil más bien sociocultural de la comunidad lingüística en estudio. En consideración a esto, para la estratificación socioeconómica, una de las variables más determinantes fue el nivel educacional y, por esta razón, es la que asigna el mayor puntaje. Asimismo, el nivel de ingresos demuestra muy poca relevancia para los estudios de mercado antes mencionados, por ello no fue considerado en la determinación de los perfiles socioeconómicos.

8 En la selección de las entrevistas del corpus se cauteló la consistencia entre las variables antes mencionadas de manera que respondieran a lo que Prieto (1995-1996), siguiendo a Lenski (1954), denominara como congruencia de estatus. 
paquete estadístico al que hemos recurrido para la estadística inferencial es el SPSS (Statistical Package for the Social Sciences), versión 15.0 para Windows, específicamente, la prueba Análisis de varianza ANOVA. En atención a que la distribución de los datos, en algunos casos, pudiera ser anormal, se complementará dicho análisis con su análogo de tipo no paramétrico, esto es, Anova de Kruskal Wallis. En ambos casos, el grado de significación se definirá en el $5 \%$, según el cual $\mathrm{p}=<0,05$ será estadísticamente significativo.

\section{Presentación y análisis de los resultados}

\subsection{Análisis discursivo de los reformuladores de rectificación relevados en el corpus}

Los marcadores de reformulación rectificativa, como ya se indicó, corrigen un segmento discursivo anterior que se considera como una formulación incorrecta. Nuestro análisis dio como resultado un total de 256 ocurrencias de reformuladores rectificativos, de acuerdo con la siguiente distribución de frecuencias absolutas del paradigma correspondiente: o sea (200), digo (7), digamos (7), más bien (4), es decir (1), bueno (29), miento (6) y perdón (2). El gráfico 1 presenta las frecuencias porcentuales de los marcadores de rectificación, en nuestros materiales:

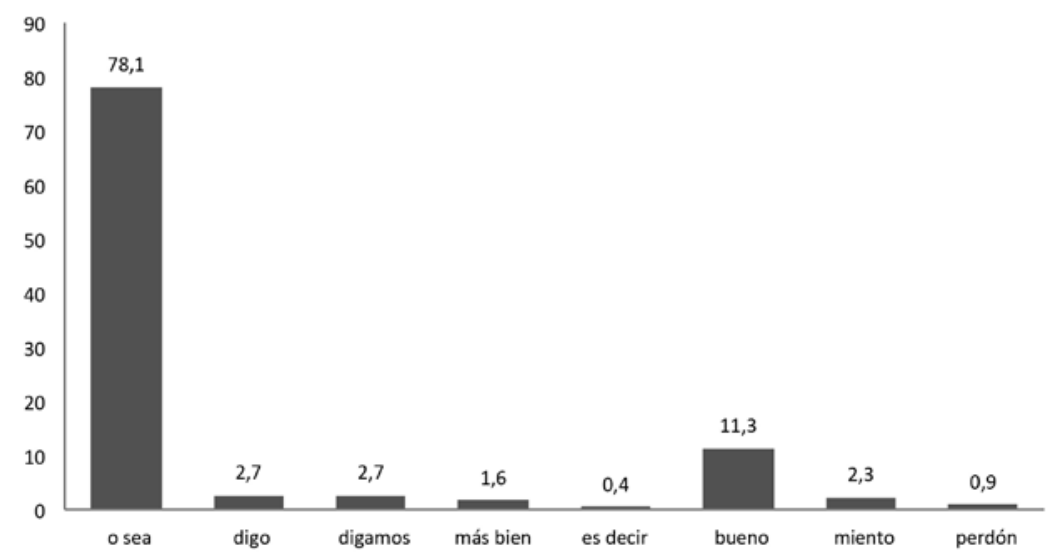

Gráfico 1. Porcentaje de frecuencia de los marcadores rectificativos en el corpus 
Por consiguiente, de acuerdo con nuestros datos, los reformuladores de rectificación más empleados en la muestra analizada son o sea y, a bastante distancia, bueno. El resto de los rectificadores manifiesta un uso significativamente menor.

Los siguientes fragmentos de las entrevistas ejemplifican el empleo de los marcadores rectificativos en el corpus analizado:

O sea

1) es un problema que es irreversible/ y las autoridades que son responsables/ que son// o sea no creo que responsables/ porque en realidad son unos hijos de puta (MAIIIM187),

2) es muy grande el porcentaje de gente mayor que hay/ y la y el el grado de de mortalidad es/o sea/ de vivencia como se dice o se podría decir es es mayor (MAIIIM185),

3) yo recuerdo haber leído el Papelucho/ muchas veces y lo volví a leer/ y lo volví a leer y al final ya me lo sabía de memoria con su hermana Domi/ o sea con la hermana Ji con la Domi que era la empleada (MIIIM139),

4) mi hijo pesaba dos kilos tres cincuenta entonces/ era todo un era muy chiquitito// y las niñitas frecuentemente no lo no lo pueden supe/ o sea las niñas lo pueden superar/ los niños// generalmente no / porque donde no está tan bien preparado como la mujer cuando nace (MBIIM076),

5) mi mamá vive en La Serena/ o sea nació en La Serena (MBIM061),

9 Respecto de la transcripción de los ejemplos, es necesario señalar las siguientes convenciones gráficas: 1) cuando corresponda a ejemplos de mayor extensión, $\mathrm{E}=$ entrevistador e I = informante; 2) como se trata de variables no fonéticas y, por lo tanto, la pronunciación no es el foco de nuestro análisis, el texto correspondiente a cada ejemplo se transcribe en ortografía convencional, incluidos los acentos gráficos; 3 ) con el propósito de no desnaturalizar demasiado la representación del habla chilena, se ha conservado la manifestación gráfica de determinados usos característicos del español hablado en Chile, como el uso del voseo paradigmático con elisión de $-s$, por ejemplo, estái, soi, viví (estáis, sois, vivís con valor de segunda persona del singular, tú) y el empleo de marcadores del discurso, como ¿cachái? ("¿entiendes?") y po (pues); 4) para la mejor comprensión de los ejemplos incluidos en este estudio, se ha suprimido el sistema de etiquetas pertenecientes al Standard Generalized Markup Language (SGML) contenidas en el Text Encoding Initiative (TEI), que se emplea en la transcripción de las entrevistas de ESECH, excepto las pausas que se señalan con /. Al final de cada ejemplo, se indica, entre paréntesis, el código del informante, según las siguientes convenciones: estrato socioeconómico ( $\mathrm{MA}=$ medio alto, $\mathrm{M}=$ medio, $\mathrm{MB}=$ medio bajo, $\mathrm{B}=$ bajo), grupo etario (III = adultos mayores de 55 años y más, II = sujetos adultos de edad intermedia entre 35 y 54 años y $\mathrm{I}=$ hablantes jóvenes de entre 20 y 34 años) y sexo $(\mathrm{M}=$ mujer y $\mathrm{H}=$ hombre $)$. A continuación del sexo se indica el número correlativo del sujeto en el corpus. 
6) nosotros estábamos aquí/ yo me levanté/ porque el niño estaba con la naricita apretada/ y me levanté a buscar unas gotitas que tenía en el baño/ cuando entro/ o sea / cuando abro la puerta de mi pieza/ está abierta hasta atrás / y el equipo lo tenían/ aquí al medio del comedor (BIIIM044),

7) no es como antes/ que antes nuestros papás huevón/ se casaron cuando teníamos / o sea cuando ellos tenían/ quince a veinte años quizás po/ y ahora se casan como a los treinta (BIH005).

Digo

8) lo celebro como lo celebra mi familia/ pero supongo que si/ algún momento estoy lejos/ no voy a celebrarlo realmente/ va a ser como/ digo lejos de mi familia/ no me llama particularmente la atención/ y si es que alguna vez llego a tener hijos / no sé cómo pensar esa cuestión de la celebración de la navidad (MAIH146),

9) E: ee ¿tú viste el el reality show de Canal 13 "Amor ciego"? I: no/ casi nada/ pero sí sabía de qué se trataba/ porque en la casa más de alguien lo veía E: ¿y qué te pareció este programa/ en general? I: pero ese lo vi po/ el segundo no lo vi / a lo/ digo/ el primero sí/ no/ el primero lo vi/ el segundo no lo vi (MIIM123).

\section{Digamos}

10) la navidad en mi familia/ bueno la verdad de las cosas que mi familia yo nunca la he pasado sola con mi familia comiendo los cuatro/ nunca/ nunca nunca nunca/ creo que/ a ver/ una vez cuando la $\mathrm{C}$ estaba como chiquitita/ pero nosotros siempre la pasamos con la familia/digamos con el familión con la familia grande/ ¿ya? (MIIIM138),

11) nosotros cuando nos casamos por el civil/ vivíamos / ya vivíamos con mi/ con mi esposo/ ya vivíamos digamos/ convivíamos (MIIM123),

12) tenemos / tengo amigos aquí entre mis vecinos y formamos con los vecinos digamos / con las vecinas / en este caso/ un centro de madres (MBIIM077).

Más bien

13) por lo tanto yo no yo no yo no le atribuyo a que el el el que que que en esta época fuertemente sea por una por una transformación/ ee 
del calentamiento o de cosas así similares/ no/ yo creo que más bien son épocas en que nuestro sistema sufre algunos trastornos y son parte de su existencia digamos (MAIIIH179),

14) yo creo que está mal enfocada// ee o sea/ no es que esté mal enfocada sino más bien yo creo que/ que el currículum que es en definitiva lo que guía el el lo que/ lo que los niños tienen que aprender en la escuela// está muy centrado en los contenidos (MIIM121).

Es decir

15) tenía alergia a los medicamentos/ es decir/ no tenía alergia a los medicamentos/ tenía una una etapa de mi vida/ de de chico que me me daba una cuestión/ que después descubrieron que se llamaba edema de glotis (MAIIH162).

Bueno

16) la navidad claro bueno la celebramos mi familia// junto a las otras digamos la familia de mi viejo y la familia de mi vieja/ y lo que hacemos es / / partimos a la casa de mis abuelos paternos / / a comer allá / comemos allá y pasamos el rato hasta más o menos las doce// para abrir regalos/ que es un poco/ bueno siempre lo hacen un poco antes porque hay harto cabro chico y son re impacientes/ (MAIH147),

17) desde chica y bueno por familia/ siempre católica/ ee pero como que se podría decir que/ bueno católica a mi manera tampoco eso no me gusta/ pero sí te podría decir que creo en Dios como alguien superior y todo/ pero ir como siempre a la iglesia/ todos los domingos/ no// o sea cuando me nace voy/ cuando no/ no (MIM108),

18) y después ya los otros cursos tuve ee que hacer sétimo y octavo en la/ bueno/ sexto y sétimo lo hice en la nocturna (BIIH020).

\section{Miento}

19) y el día lunes en la noche/ me voy a acostar / fui al colegio y todo/ y el día lunes en la noche / ya me voy a acostar / / lego del colegio/ no/ miento/ no estaba en el colegio/ estaba estaba trabajando ya en la papelera/ y / y mi hermano estaba en el colegio/ entonces 
el día martes yo tenía que/ yo entraba a las ocho a la papelera (MBIH053),

20) claro/ pero eso fue cuando estaba/ yo viviendo con la J po/ con mi hermana/ que cuando el $\mathrm{P}$ tenía meses nomás po/ pero cuando yo vi llegué a vivir aquí ya el P chico tenía más / como dos años / no/ miento / el P chico tenía como cuatro años más o menos ya cuando yo me vine a vivir acá// sí// o tres / como tres años aproximado porque yo/ cuando el P tenía cuatro años yo me casé (BIIM028).

Perdón

21) entonces es re divertido porque la directiva de las niñas son las niñas de diez/ no/ desde kin de pre kin/ perdón desde primer / hasta sexto/ entonces las chiquititas de primero ¡son tan divertidas! (MAIIM172).

Como se desprende de los ejemplos 1 al 21, los marcadores rectificativos introducen un miembro que corrige el contenido formulado previamente. El marcador o sea es, por lejos, el más frecuente en la categoría de los rectificativos, si bien su función preeminente es las de reformulador explicativo. A este respecto, Martín Zorraquino y Portolés (1999) señalan que, además de los reformuladores específicos de esta categoría, o sea también puede utilizarse como rectificador. Otros reformuladores del corpus que también asumen un valor polifuncional junto a o sea, asumiendo -en ocasiones- un valor rectificativo, son digamos y es decir, que mayormente también se comportan como explicativos en los materiales analizados. En las clasificaciones de marcadores del discurso consultadas se consigna a digo y más bien con un valor rectificativo ya convencionalizado; sin embargo, llama la atención que su empleo sea tan restringido en la muestra. También relevamos un significado rectificativo de bueno, el que -como veremos-constituye un marcador que asume múltiples funciones en la muestra. Finalmente, por lo que respecta a miento y perdón, se trata de marcadores no plenamente gramaticalizados, pero que parecen estar motivados por el cumplimiento de la máxima conversacional de calidad de Grice (1975). El Diccionario de la lengua española registra una acepción como interjección de miento "para corregirse alguien a sí mismo cuando advierte que ha errado o se ha equivocado" (RAE, 2001, s. v. mentir). Con respecto a perdón, es 
posible que sea un uso más idiosincrásico del español del Chile, en el que también se percibe que los hablantes son, hasta cierto punto, conscientes del incumplimiento de una determinada regla de comportamiento ${ }^{10}$.

Por otra parte, así como ocurrió con otros marcadores del corpus, una de las tareas del análisis fue discriminar los usos no rectificativos del marcador bueno, el que mostró más de una función discursiva en el corpus. Los fragmentos que a continuación comentamos muestran valores discursivo-pragmáticos de bueno diferentes a la reformulación rectificativa:

22)E: ee ¿de qué forma podrían atribuirse tales cambios al llamado calentamiento global/ al efecto invernadero o al fenómeno del niño o la niña? I: ee/ bueno/ no cacho mucho de esas huevadas pero// como por lo que he visto y lo que he/ bueno no no diría lo que he investigado/ porque yo no investigo esas cosas/ pero lo lo lo lo que he cachado como que se atribuye más al calentamiento global/ creo que están en distintos lugares del mundo produciéndose cambios climáticos muy extraños ¿cachái? (MAIH147),

23) cuando estaba más chica y todavía no tenía a mi hijo// puta un carrete memorable fue/ una vez que fui al cumpleaños de un amigo y bueno ahí tomé caleta/ pucha ahí tomé cerveza/ pisco/ ron// todo lo que había tomé/ y más encima después al cumpleañero/ le regalaron un tequila y con ese tequila nos pusimos a tomar tequila/ y yo me tomé cinco golpeaditos ¿cachái? / y ese y ese tequila me mató/ ¿cachái? que voy con unas amigas / y de repente veo/ / bueno ahí pierdo la noción / y me veo en el baño vomitando// de lo único que me acuerdo/ en el baño vomitando/ y después que yo juraba que habían pasado como quince minutos y había pasado como una hora// y mis amigas se turnaban para cuidarme mientras yo vomitaba/ después me fueron a acostar/ bueno yo caminé/ obviamente/ había tanta

10 Con respecto a miento y perdón, nos parece interesante la posibilidad de comprobar el funcionamiento del Principio de Cooperación y la Máxima de Calidad de Grice (1975), a partir del análisis de los marcadores del discurso. Asimismo, nos llama la atención que miento no aparezca consignado en las clasificaciones de marcadores más conocidas, considerando las características de la definición de la RAE (2001) antes citada. 
gente que me tuve que tirar en el suelo a dormir / y estaba muerta de frío / al otro día nos fuimos muy temprano y yo iba con la caña viva en la micro/ me sentía pésimo (MIM108).

En 22, bueno se emplea como introductor de respuesta, mientras en 23 funciona como marcador continuativo que favorece la ilación narrativa del relato de la hablante. Nótese, además, que en 22, a continuación de introducir su respuesta, el entrevistado ocupa bueno como rectificativo para autocorregirse.

\section{2. Análisis sociolingüístico de los reformuladores de rectificación relevados en el corpus}

En esta sección, se presentan los resultados de nuestro estudio respecto de la relación de la frecuencia de empleo de los reformuladores de rectificación con las tres variables extralingüísticas consideradas en el análisis: sexo, edad y grupo socioeconómico de los sujetos de la muestra. Según el criterio invocado anteriormente, las pruebas de estadística inferencial se aplicarán solo a los rectificativos o sea y bueno, en virtud de que superan los 25 casos.

\section{2. 1. Sexo}

En la tabla 2 se muestran las frecuencias absoluta y porcentual de la ocurrencia de los reformuladores de rectificación identificados en el corpus, según el sexo de los sujetos.

\begin{tabular}{lll}
\hline Marcadores & Hombres & Mujeres \\
\hline o sea & $89(77,4)$ & $111(78,7)$ \\
\hline digo & $3(2,6)$ & $4(2,8)$ \\
\hline digamos & $3(2,6)$ & $4(2,8)$ \\
\hline más bien & $2(1,7)$ & $2(1,4)$ \\
\hline es decir & $1(1)$ & $0(0)$ \\
\hline bueno & $12(10,4)$ & $17(12)$ \\
\hline miento & $5(4,3)$ & $1(0,9)$ \\
\hline perdón & $0(0)$ & $2(1,4)$ \\
\hline Totales & $115(100)$ & $141(100)$ \\
\hline
\end{tabular}

Tabla 2. Frecuencia absoluta y porcentaje de los marcadores de reformulación rectificativa relevados en el corpus según el sexo de los sujetos de la muestra 
En relación con el análisis de las medias correspondientes a la ocurrencia de o sea como reformulador rectificativo según el factor sexo de los sujetos, el gráfico 2 muestra los resultados del análisis paramétrico, según el cual la media para los hombres es 1,483, mientras que la de las mujeres alcanza a 1,850. No obstante, los resultados no son significativos estadísticamente $(\mathrm{F}=1,024, \mathrm{p}=0,314)$.

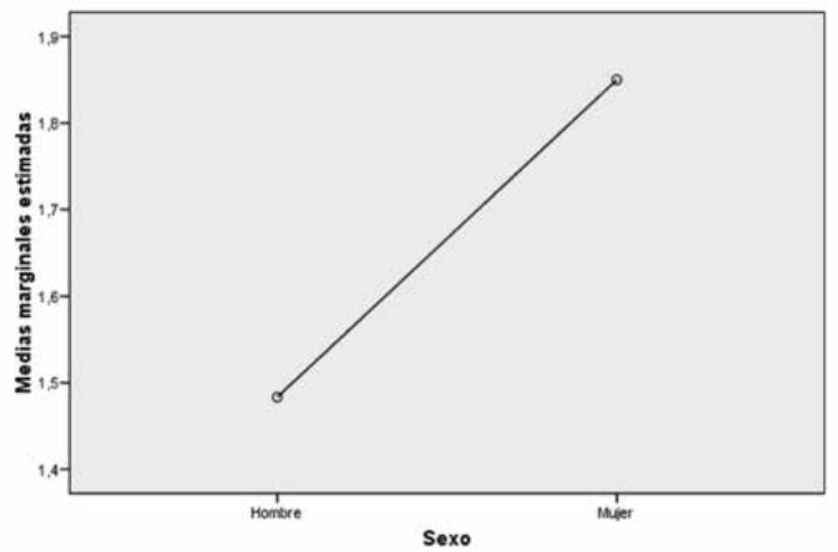

Gráfico 2. Medias marginales estimadas de o sea rectificativo según sexo

La aplicación de la prueba no paramétrica Anova de Kruskal-Wallis, por su parte, arroja como rangos promedio para los hombres el de 57,21y para las mujeres el de 63,79. Sin embargo, el mismo análisis descarta la significatividad estadística de dichas diferencias, ya que Chi-cuadrado= $1,148, \mathrm{p}=0,284$.

Por otro lado, la varianza del marcador rectificativo bueno (gráfico 3) especifica las siguientes diferencias entre las medias y el sexo de los sujetos: hombres $(0,200)$ y mujeres $(0,283)$, donde $F=0,537, p=0,465$, por lo tanto, se trata de diferencias no significativas. 


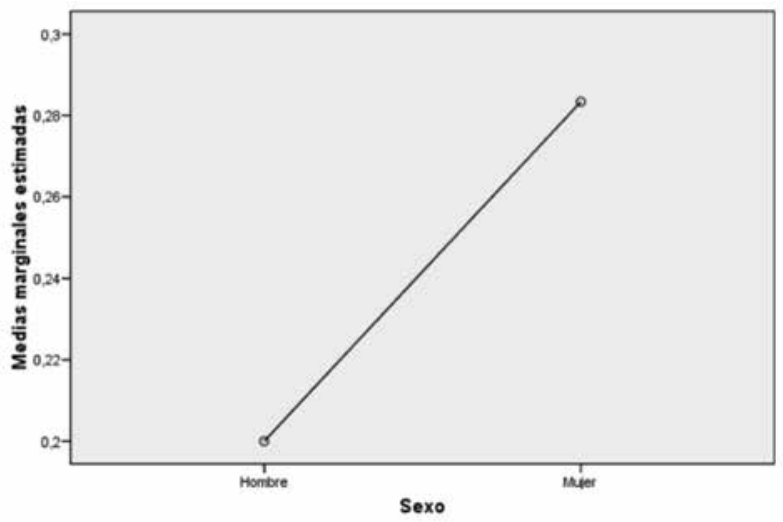

Gráfico 3. Medias marginales estimadas de bueno según sexo

La aplicación de la prueba no paramétrica, por su parte, arroja como rangos promedio para los hombres el de 59,45 y para las mujeres el de 61,55. Sin embargo, el mismo análisis descarta la significatividad estadística de dichas diferencias, ya que Chi-cuadrado=0,260, $\mathrm{p}=0,610$.

\section{2. 2. Edad}

La tabla 3 expone las frecuencias absoluta y porcentual de la ocurrencia de los marcadores de reformulación rectificativa relevados en el corpus, de acuerdo con la edad de los sujetos.

\begin{tabular}{llll}
\hline Marcadores & 20 a 34 & 35 a 54 & 55 y más \\
\hline o sea & $93(84,5)$ & $54(67,5)$ & $53(80,3)$ \\
\hline digo & $3(2,7)$ & $3(3,8)$ & $1(1,5)$ \\
\hline digamos & $0(0)$ & $3(3,8)$ & $4(6,1)$ \\
\hline más bien & $0(0)$ & $3(3,8)$ & $1(1,5)$ \\
\hline es decir & $0(0)$ & $1(1,1)$ & $0(0)$ \\
\hline bueno & $13(11,8)$ & $10(12,5)$ & $6(9,1)$ \\
\hline miento & $1(1)$ & $4(5)$ & $1(1,5)$ \\
\hline perdón & $0(0)$ & $2(2,5)$ & $0(0)$ \\
\hline Totales & $110(100)$ & $80(100)$ & $66(100)$ \\
\hline
\end{tabular}

Tabla 3. Frecuencia absoluta y porcentaje de los marcadores de reformulación rectificativa relevados en el corpus según la edad de los sujetos de la muestra 
El gráfico 4 presenta los resultados de la comparación de las medias correspondientes a o sea rectificativo, de acuerdo con la edad de los hablantes de la muestra. La media para el primer grupo (20-34 años) es de 2,325, mientras que para el segundo (35-54) de 1,350 y para el tercero (55 y más) de 1,325. Estas diferencias son estadísticamente significativas, ya que $\mathrm{F}=3,438, \mathrm{p}=0,035$.

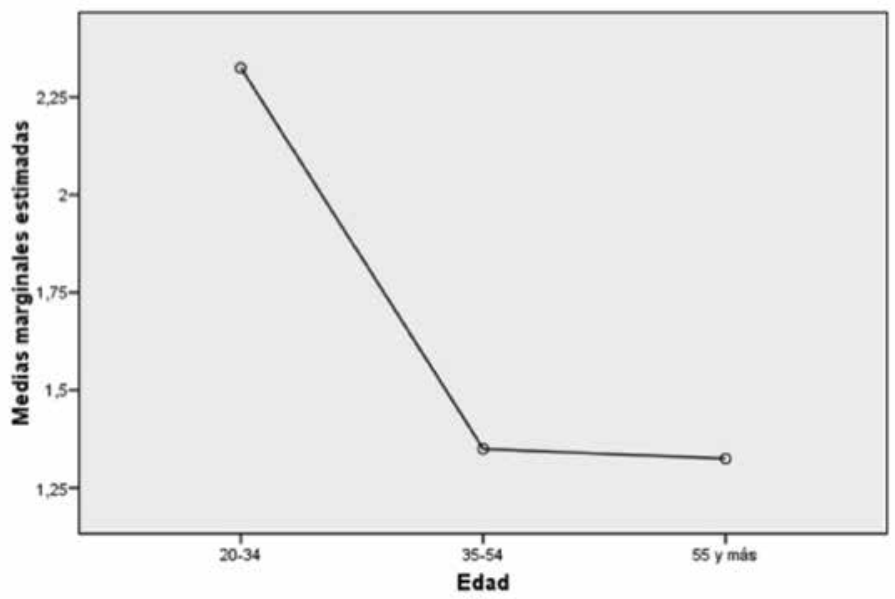

Gráfico 4. Medias marginales estimadas de o sea rectificativo según edad

La prueba Anova de Kruskal-Wallis, por su parte, da los siguientes rangos promedio para las edades de los sujetos: $20-34$ años (71,86), 35-54 $(54,45)$ y 55 y más $(55,19)$. La misma prueba confirma que estas diferencias son significativas, puesto que Chi-cuadrado $=6,847, \mathrm{p}=0,033$.

En relación con el análisis de las medias correspondientes a la ocurrencia de bueno como reformulador rectificativo según la edad de los sujetos, el gráfico 5 muestra los resultados del análisis paramétrico, según el cual las medias para los grupos etarios son: 20-34 (0,325), 35-54 $(0,250)$ y 55 y más $(0,150)$. No obstante, los resultados no son significativos estadísticamente $(\mathrm{F}=0,795, \mathrm{p}=0,454)$. 


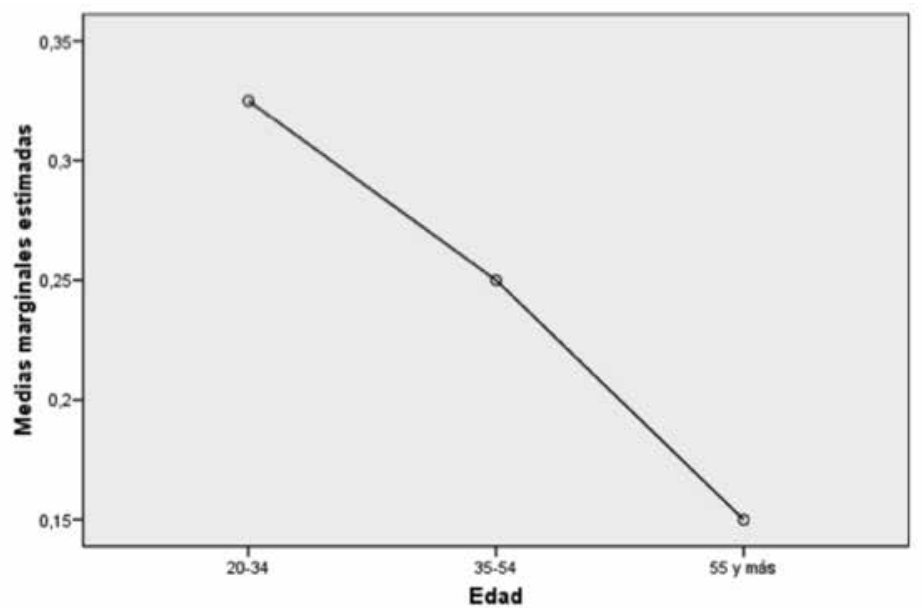

Gráfico 5. Medias marginales estimadas de bueno según edad

La aplicación de la prueba no paramétrica Anova de Kruskal-Wallis, por su parte, arroja como rangos promedio para los grupos de edad: 20$34(66,51), 35-54(58,36)$ y 55 y más $(56,63)$. Sin embargo, el mismo análisis descarta la significatividad estadística de dichas diferencias, ya que Chicuadrado $=4,388, \mathrm{p}=0,111$.

\section{2. 3. Grupo socioeconómico}

En la tabla 4 se muestran las frecuencias absoluta y porcentual de la ocurrencia de los reformuladores de rectificación identificados en el corpus, según el grupo socioeconómico de los sujetos.

\begin{tabular}{lllll}
\hline Marcadores & $\mathrm{B}$ & $\mathrm{MB}$ & $\mathrm{M}$ & $\mathrm{MA}$ \\
\hline o sea & $47(82,5)$ & $48(90,1)$ & $35(68,6)$ & $70(73,7)$ \\
\hline digo & $0(0)$ & $0(0)$ & $3(5,9)$ & $4(4,2)$ \\
\hline digamos & $0(0)$ & $4(7,5)$ & $2(3,9)$ & $1(1,1)$ \\
\hline más bien & $1(1,7)$ & $0(0)$ & $1(2)$ & $2(2)$ \\
\hline es decir & $0(0)$ & $0(0)$ & $0(0)$ & $1(1,1)$ \\
\hline bueno & $4(7)$ & $0(0)$ & $9(17,6)$ & $16(16,8)$ \\
\hline miento & $5(8,8)$ & $1(2,4)$ & $0(0)$ & $0(0)$ \\
\hline perdón & $0(0)$ & $0(0)$ & $1(2)$ & $1(1,1)$ \\
\hline Totales & $57(100)$ & $53(100)$ & $51(100)$ & $95(100)$ \\
\hline
\end{tabular}

Tabla 4. Frecuencia absoluta y porcentaje de los marcadores de reformulación rectificativa relevados en el corpus según el grupo socioeconómico de los sujetos de la muestra 
La comparación de las medias correspondientes a la frecuencia de o sea rectificativo, según el grupo socioeconómico de los sujetos, se expone en el gráfico 6. La prueba ANOVA proporciona los siguientes resultados: bajo $(1,567)$, medio bajo $(1,600)$, medio $(1,167)$ y medio alto $(2,333)$. Sin embargo, según dicho procedimiento paramétrico, los resultados no son significativos estadísticamente, puesto que $\mathrm{F}=1,838, \mathrm{p}=0,144$.

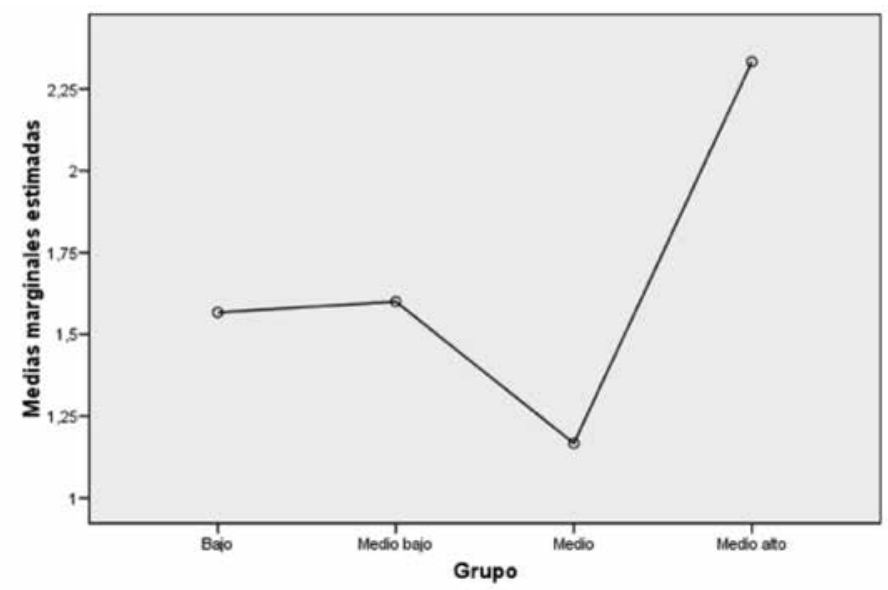

Gráfico 6. Medias marginales estimadas de o sea rectificativo según grupo socioeconómico

De igual modo, la prueba no paramétrica seleccionada entrega los siguientes rangos promedio para grupos socioeconómicos de los sujetos: bajo $(60,68)$, medio bajo $(59,65)$, medio $(52,88)$, medio alto $(68,78)$. Consecuentemente, dicha prueba confirma que estas diferencias no son significativas, ya que Chi-cuadrado=3,373, $\mathrm{p}=0,338$.

Por lo que se refiere a la comparación de las medias correspondientes a la ocurrencia de bueno como reformulador de rectificación, según el grupo socioeconómico de los sujetos, el gráfico 7 muestra los resultados del análisis paramétrico ANOVA, según el cual las medias para los grupos son: bajo $(0,133)$, medio bajo $(0,000)$, medio $(0,300)$ y medio alto $(0,533)$. De igual manera, estas diferencias entre las medias de los grupos son estadísticamente significativas $(\mathrm{F}=4,462, \mathrm{p}=0,005)$. 


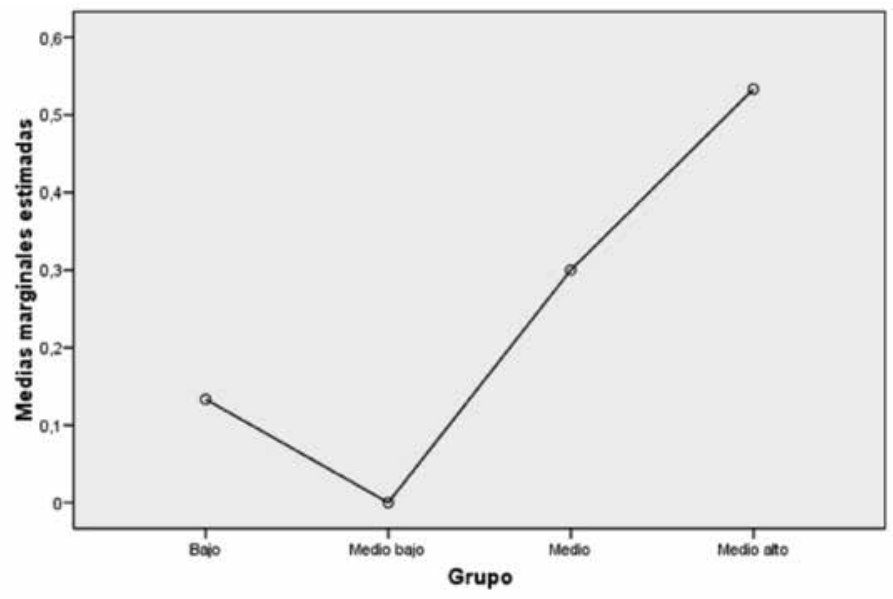

Gráfico 7. Medias marginales estimadas de bueno según grupo socioeconómico

La prueba no paramétrica de Kruskal-Wallis confirma de modo consecuente la significatividad de estas diferencias, puesto que Chicuadrado $=13,888, p=0,003$. Además, señala los siguientes rangos promedio para los grupos de la muestra: bajo $(56,47)$, medio bajo $(50,50)$, medio $(64,32)$ y medio alto $(70,72)$.

En síntesis, las diferencias de edad de los hablantes resultaron significativas en el empleo de o sea como reformulador rectificativo, ya que se trata de un uso preponderante entre los hablantes jóvenes de la comunidad en estudio. De igual manera, se llega a un resultado significativo, estadísticamente, con respecto a las diferencias entre los grupos socioeconómicos en el uso de bueno como marcador de rectificación, puesto que su frecuencia es mayor entre los hablantes del grupo medio alto.

\section{Conclusiones}

Los hallazgos más relevantes de este estudio pueden resumirse como sigue:

1. En nuestros materiales se identificaron 256 ocurrencias de la función discursiva (variable) "reformulación rectificativa mediante marcadores", la que se materializó por medio del siguiente 
paradigma de rectificadores (variantes), con las frecuencias absolutas y porcentuales que se indican: o sea $(200=78,1 \%)$, digo $(7=2,7 \%)$, digamos $(7=2,7 \%)$, más bien $(4=1,6 \%)$, es decir $(1=0,4 \%)$, bueno $(29=$ $11,3 \%)$, miento $(6=2,3 \%)$ y perdón $(2=0,9 \%)$.

2. Los factores sociodemográficos más sensibles al empleo de los reformuladores de rectificación fueron, en orden de importancia, la edad y el grupo socioeconómico de los informantes. No se encontraron diferencias de sexo notorias, por lo que el mencionado factor resultó ser intrascendente desde el punto de vista estadístico.

3. Por lo que se refiere a los rectificadores más frecuentes, el empleo de o sea está más extendido entre los hablantes del primer grupo etario (20 a 34 años), mientras que el de bueno lo está entre los del grupo socioeconómico medio alto. Estas diferencias de uso de los mencionados rectificadores resultaron ser significativas en términos estadísticos.

4. La polifuncionalidad de algunos rectificadores nos ha permitido comprobar las ventajas de la orientación onomasiológica que hemos adoptado en nuestro análisis; esto es, identificar una función discursiva básica común, en términos de instrucciones semánticas, y aplicar la prueba de la conmutación (Portolés, 2001, p. 79-84), con el objetivo de determinar cuáles son los marcadores que -formalmentela cumplen en el discurso.

5. Para finalizar, quisiéramos insistir en el carácter naturalmente relativo de los resultados de este trabajo, ya que se limita a describir el uso de un tipo específico de marcador del discurso en una comunidad de habla española en particular. En este sentido, resultaría interesante llevar a cabo trabajos contrastivos con muestras de otras comunidades de habla española que replicaran lo desarrollado en la presente estudio de manera de corroborar o relativizar sus resultados. De igual modo, sería pertinente ampliar un análisis pragmático y sociolingüístico como el que aquí aplicamos a otros tipos de marcadores del discurso en el habla de Santiago de Chile. 


\section{Referencias bibliográficas}

ADIMARK (Investigaciones de Mercado y de Opinión Pública). (2003). Mapa socioeconómico de Chile. Nivel socioeconómico de los hogares del país basado en datos del Censo. Recuperado de: http: / /www.adimark.cl/medios / estudios/mapa_socioeconomico_de_chile.pdf

AIM (Asociación de Investigadores de Mercado). (2008). Grupos socioeconómicos 2008. Recuperado de: http: / / www.anda.cl/estudios / textos / DescripcionGSEChile2008.pdf

Anscombre, J. y O. Ducrot. (1994). La Argumentación en la lengua. Madrid: Gredos.

Carbonero, P. y J. Santana. (2010). Marcadores del discurso, variación dialectal y variación social. En Loureda, Ó. y E. Acín (Coords.), Los estudios sobre marcadores del discurso, hoy (pp. 497-521). Madrid: Arco Libros.

Cortés, L. (1998). Marcadores del discurso y análisis cuantitativo. En M. Martín Zorraquino y E. Durán (Coords.), Los marcadores del discurso. Teoría y análisis (pp. 143160). Madrid: Arco/ Libros.

Cuenca, M. J. (2003). Two ways to reformulate: a contrastiveanalysis of reformulation markers. Journal of Pragmatics, 35, 1069-1093.

Garcés Gómez, M. P. (2003). Los marcadores de recapitulación y de reconsideración en el discurso. Revista de Investigación Lingüística, 1 (6), 111-141.

(2008). La organización del discurso: marcadores de ordenación y reformulación. Madrid/Frankfurt: Iberoamericana/ Vervuert.

- (2010). Marcadores de corrección y rectificación en los textos escritos. Revista de Investigación Lingüística, 13, 87105.

. (2011). Procesos de reformulación en el discurso oral: corrección y rectificación. Español Actual, 96, 89-106. 
Grice, P. (1975). Logic and conversation. En P. Cole y J. Morgan (eds.), Speech and semantics, 3: Speech acts (pp. 41- 58). Nueva York: Academic Press.

Gülich, E.y T. Kotschi. (1983). Les marqueurs de reformulation paraphrastique. Cahiers de Linguistique Française, 5, 305-351.

(1995). Discourse Production in Oral Communication. A Study Based on French. En U. Quasthoff (Ed.), Aspects of Oral Communication (pp. 30-66). Berlín: De Gruyter.

Hernández Campoy, J. y M. Almeida. (2005). Metodología de la investigación sociolingüística. Málaga: Editorial Comares.

ICCOM (Instituto Consultor en Comercialización y Mercado). (2005). Descripción Básica de los Niveles Sociales Hogares Urbanos Región Metropolitana. Recuperado de:

http: / / www.cadem.cl/v1/ files/estadistico/descripcionBasica_GSE_ ICCOM_2005.pdf

Labov, W. (1983). Modelos sociolingüísticos. Madrid: Cátedra.

Lavandera, B. (1978). Where does the sociolinguistic variable stop? Language in Society, 7, 171-182.

Lenski, G. (1954). Status Crystallization: A Non-vertical Dimension of Social Status. American Sociological Review, 19, 405413.

López Morales, Humberto. (1994). Métodos de investigación lingüística. Salamanca: Ediciones Colegio de España.

Loureda, Ó. y E. Acín (coords.). (2010). Los estudios sobre marcadores del discurso, hoy. Madrid: Arco Libros.

Martín Zorraquino, M. (1998). Los marcadores del discurso desde el punto de vista gramatical. En M. Martín Zorraquino y E. Montolío (Coords.), Los marcadores del discurso. Teoría y análisis (pp. 19-53). Madrid: Arco/Libros.

Martín Zorraquino, M. y J. Portolés. (1999). Los marcadores del discurso. En I. Bosque y V. Demonte (Eds.), Gramática 
descriptiva de la lengua española (pp. 4051-4207). Madrid: Espasa Calpe.

Moreno Fernández, F. (1998). Principios de sociolingüística y sociología del lenguaje. Barcelona: Ariel. . (1990). Metodología sociolingüística. Madrid: Editorial Gredos.

Portolés, J. (2001). Marcadores del discurso. Barcelona: Ariel. (2004). Pragmática para hispanistas. Madrid: Síntesis.

Prieto, L. (1995-1996). Análisis sociolingüístico del dequeísmo en el habla de Santiago de Chile. Boletín de Filología, XXXV, 379 -452 .

RAE (Real Academia Española). (2001). Diccionario de la lengua española. (22 ${ }^{\mathrm{a}}$ ed.). Madrid: Espasa Calpe.

Rojas, C., A. Rubio, A. San Martín y S. Guerrero. (2012). Análisis pragmático y sociolingüístico del empleo de los marcadores discursivos de reformulación en el habla de Santiago de Chile. Lenguas Modernas, 40, 103-123.

Rossari, C. (1994). Les operations de reformulation. Berna: Peter Lang.

San Martín, A. (2004-2005). Igual como marcador discursivo en el habla de Santiago de Chile: función pragmático-discursiva y estratificación social de su empleo. Boletín de Filología, XL, $201-232$.

(2011). Los marcadores interrogativos de control de contacto en el corpus PRESEEA de Santiago de Chile. Boletin de Filología, XLVI (2), 135-166.

. (2013). Los reformuladores de distanciamiento en el corpus PRESEEA de Santiago de Chile. Boletín de Filología, XLVIII (1), 171-199.

Serrano, M. J. (2011). Sociolingüística. Barcelona: Ediciones del Serbal.

Silva-Corvalán, C. (2002). Sociolingüística y pragmática del español. Washington: Georgetown University Press.

Schiffrin, D. (1987). Discourse Markers. Cambridge: Cambridge University Press. 
(2003). Discourse Markers: Language, Meaning and Context. The Handbook of Discourse Analysis (pp. 54-75). Oxford: Blackwell.

Trudgill, P. y J. Hernández Campoy. (2007). Diccionario de sociolingüística. Madrid: Gredos.

Valencia, A. (2014a). Introducción. Cuadernos de la ALFAL, 5, 4-12.

Valencia, A. (2014b). Marcadores del discurso en Santiago de Chile. Cuadernos de la ALFAL, 5, 246-276. 